\title{
Electron loss mechanisms in a miniature microwave discharge water neutralizer
}

Cite as: Phys. Plasmas 27, 063505 (2020); https://doi.org/10.1063/5.0002336

Submitted: 23 January 2020 . Accepted: 11 May 2020 . Published Online: 01 June 2020

Yosuke Sato, Hiroyuki Koizumi (D), Masakatsu Nakano, and Yoshinori Takao (D)

\section{Sign up for topic alerts}

New articles delivered to your inbox 


\title{
Electron loss mechanisms in a miniature microwave discharge water neutralizer
}

\author{
Cite as: Phys. Plasmas 27, 063505 (2020); doi: 10.1063/5.0002336 \\ Submitted: 23 January 2020 . Accepted: 11 May 2020 . \\ Published Online: 1 June 2020
}

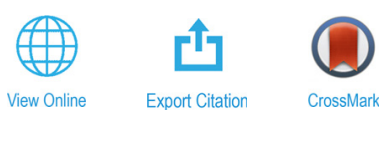

\author{
Yosuke Sato, ${ }^{7}$ Hiroyuki Koizumi, ${ }^{2}$ (D) Masakatsu Nakano, ${ }^{3}$ and Yoshinori Takao ${ }^{4, a)}$ (D)
}

\begin{abstract}
AFFILIATIONS
'Department of Mechanical Engineering, Materials Science, and Ocean Engineering, Yokohama National University, Yokohama 240-8501, Japan

${ }^{2}$ Department of Advanced Energy, The University of Tokyo, Kashiwa, Chiba 277-8561, Japan

${ }^{3}$ Department of Engineering, Tokyo Metropolitan College of Industrial Technology, 8-17-1, Minami-Senju, Arakawa-ku, Tokyo 113-0852, Japan

${ }^{4}$ Division of Systems Research, Yokohama National University, Yokohama 240-8501, Japan
\end{abstract}

a) Author to whom correspondence should be addressed: takao@ynu.ac.jp

\begin{abstract}
This study analyzes the mechanism of electron loss at the discharge chamber wall of a microwave discharge neutralizer via three-dimensional particle-in-cell simulations with Monte Carlo collisions (PIC-MCCs). The neutralizer employs electron cyclotron resonance discharges with two ring-shaped permanent magnets and 4.2-GHz microwaves, where the plasma is confined by a magnetic mirror. The PIC-MCC simulation results show that the electron extraction efficiency of a water neutralizer can be increased by two times in an optimized magnetic field configuration, which is a higher increased rate than that of a xenon neutralizer. However, the efficiency of $20 \%$ is still low (e.g., less than half of the xenon one) because many electrons are lost to the magnet surface. The loss is determined to be due to approximately 5-times higher ratio of electrons inside the loss cone in the water neutralizer than that in the xenon neutralizer. The electron velocity distributions of each neutralizer clearly show that the water neutralizer has a larger fraction of electrons parallel to the magnetic field than the xenon neutralizer. This result is attributed to the large number of electron collisions in the water neutralizer owing to the high neutral gas pressure.
\end{abstract}

Published under license by AIP Publishing. https://doi.org/10.1063/5.0002336

\section{INTRODUCTION}

Plasma discharges of water vapor can be potentially used in a wide range of applications such as environmental and health problems, ${ }^{1-5}$ plasma-assisted chemical vapor deposition, ${ }^{6}$ and space propulsion. $^{7-9}$ In space propulsion, several types of thrusters that use water plasma have been researched and developed such as a pulsed plasma thruster, ${ }^{7}$ a helicon thruster, ${ }^{8}$ and an electrothermal thruster. ${ }^{9}$ Compared to other propellants, water propellant has several advantages such as safety, easy handling, and low manufacturing cost. Moreover, liquid propellant can considerably miniaturize a propulsion system by removing a large highpressure gas storage system, which occupies most of the weight and volume of the propulsion system. Because the demand for propulsion systems for a $10-\mathrm{kg}$-class spacecraft is considerably increasing, ${ }^{10}$ water is a promising propellant for microspacecraft.

As one of the propulsion systems for a $10-\mathrm{kg}$-class spacecraft to produce high delta- $v$ and specific impulse (fuel efficiency), a miniature microwave discharge water ion thruster has been proposed and developed by the University of Tokyo, which employs electron cyclotron resonance (ECR) discharges for its ion source and neutralizer. The water ion thruster has already operated on the ground, and its thrust performance has been measured. ${ }^{11,12}$ The measurements showed that the highest thrust and specific impulse were $164 \mu \mathrm{N}$ and $665 \mathrm{~s}$ without the neutralizer, respectively, where the thrust correction factor was determined to be 0.92 with a beam acceleration voltage of over $0.80 \mathrm{kV}$. In addition, previous experiments have indicated that the water neutralizer needs almost the same mass flow rate as its ion source for neutralization owing to the small ionization cross section and light mass of water molecules, ${ }^{12}$ whereas the xenon neutralizer needs a half or less mass flow rate of its ion source. ${ }^{13,14}$ This high consumption of the propellant in the water neutralizer is a critical issue for space propulsion systems because the propellant cannot be reloaded in space.

Although it is important to investigate the plasma behavior to increase the water neutralizer performance, the use of plasma diagnostics is difficult owing to the small size of its discharge chamber $(20 \mathrm{~mm}$ in diameter and $4 \mathrm{~mm}$ in height). To address this issue, we conducted 
three-dimensional (3D) fully kinetic particle simulations to analyze the degradation of the neutralizer performance. Here, we employed the real electron to ion mass ratio without introducing an artificial mass ratio to capture the physics of electron motion. In a previous study, ${ }^{15} 3 \mathrm{D}$ particle simulations revealed that the electron extraction efficiency, which is defined as the ratio of the extracted electron current to the generated electron current, achieved a 1.5-times higher value in an optimized magnetic field configuration, where the magnetic field lines passed through nearly the entire area of the orifices. In this study, we attempted to improve the extraction efficiency for the water neutralizer in a similar way.

In the following section (Sec. II), the numerical model is briefly described. The simulation results indicated that the electron extraction efficiency of the water neutralizer was able to be increased by two times in the optimized magnetic configuration; however, the efficiency was still less than $20 \%$ owing to the large loss of electrons to the magnet surface. The loss mechanisms are discussed in Sec. III from the viewpoint of electron velocity distribution functions. Finally, conclusions are made in Sec. IV.

\section{NUMERICAL MODEL \\ A. Calculation}

We employed 3D particle-in-cell simulations with the Monte Carlo collision (PIC-MCC) algorithm for the kinetics of charged particles, a finite-difference time-domain (FDTD) algorithm for the electromagnetic fields of microwaves, and a finite-element analysis using ANSYS $®$ Academic Teaching Mechanical and CFD, Release 15.0 for the magnetostatic fields of permanent magnets. Figure 1 shows the flow chart of the simulation used in this study. The 3D PIC-MCC simulations were based on our previous papers. ${ }^{15-19}$ First, we set the initial conditions; then, we solved Maxwell's equations using the FDTD algorithm for the electromagnetic fields of the microwaves to obtain a steady-state solution without plasma. Second, we conducted electrostatic PIC-MCC simulations using the time-varying electric field of microwaves calculated by the FDTD algorithm, the electrostatic electric field of plasma, and the magnetic fields produced by permanent magnets. In this calculation, the magnetic fields of microwaves are not taken into account because those are negligibly small compared to the magnetostatic fields of permanent magnets.

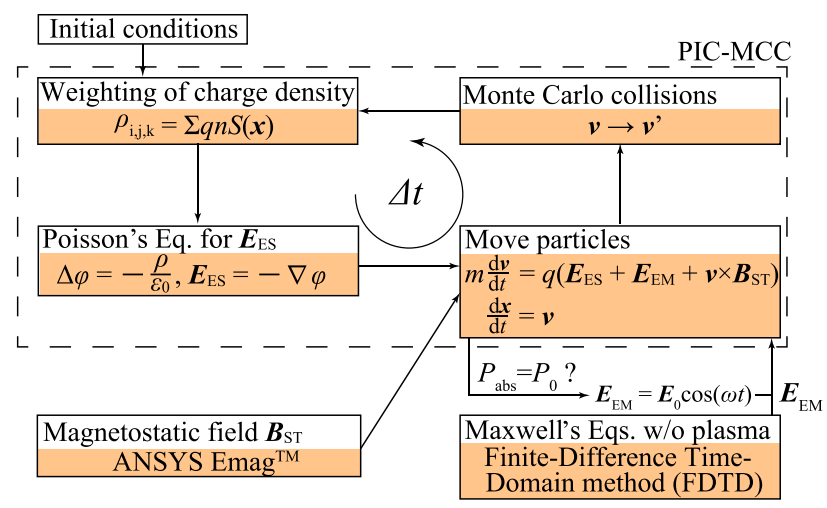

FIG. 1. Calculation flow for the PIC-MCC simulation.
For the plasma parameter range calculated in this study, the grid spacing in the PIC-MCC simulation was set to $0.1 \mathrm{~mm}$ at regular intervals in all axial directions in the entire computational domain, which is sufficiently small to satisfy the condition: the Debye length is approximately larger than a third of grid spacing. ${ }^{20}$ On the basis of grid spacing, the time increment of FDTD, $\Delta t_{\mathrm{EM}}$, was set to $1.49 \times 10^{-13} \mathrm{~s}$ to satisfy the Courant-Friedrichs-Lewy (CFL) condition, which was equal to $1 / 1600$ of a $4.2-\mathrm{GHz}$ microwave cycle employed in this study. The time steps for PIC were $\Delta t_{\mathrm{e}}=5.95 \times 10^{-12} \mathrm{~s}$ (1/40 of a microwave cycle) for the electrons and $\Delta t_{\mathrm{i}}=2.38 \times 10^{-10} \mathrm{~s}$ (one microwave cycle) for the ions, where $\Delta t_{\mathrm{e}}$ and $\Delta t_{\mathrm{i}}$ are sufficient to resolve the electron and ion plasma frequencies, respectively, and are much smaller than the mean-free time. Here, all time steps were fixed throughout the simulation. Because it is impossible to trace the motion of all charged particles, the superparticles of ions and electrons were introduced, where a single superparticle had approximately $1.0 \times 10^{4}$ of real charge and mass. Last, we rescaled the amplitude of the electromagnetic field to adjust the power absorbed in the plasma, $P_{\mathrm{abs}}$, to the input parameter of $P_{0}$. The above-mentioned procedure was iterated until a steady-state solution was obtained.

\section{B. Configurations and conditions}

Figure 2 shows a schematic diagram of the calculation model for the neutralizer together with the magnetic fields produced by two ring-shaped permanent magnets. The red lines in Fig. 2(a) indicate the resonant magnetic field of $0.15 \mathrm{~T}$, which is required to obtain ECR discharge heating for $4.2-\mathrm{GHz}$ microwaves. Here, the electrons generated by ECR heating are also confined by the magnetic mirror, and most electrons toward the upstream surface are lost to the permanent magnet. Figure 2(a) shows two magnetic field configurations, which are referred to as MF-1 and MF-2; MF-1 is the conventional magnetic field used in previous papers, ${ }^{16-19}$ and MF-2 is the optimized magnetic configuration, which has a smaller outer diameter of the inner ringshaped permanent magnet. To maintain distance between the ECR layer and the antenna, the gap distance from the magnet surface to the antenna for MF-2 is $0.3 \mathrm{~mm}$, whereas that for MF-1 is $0.8 \mathrm{~mm}$. In the previous study, the electron extraction efficiency of the xenon neutralizer with MF-2 was increased by 1.5 times. ${ }^{15}$ Hence, in this study, we also conducted simulations for the water neutralizer with MF-1 and MF-2 and compared the electron extraction efficiency.

A Cartesian coordinate system is employed in PIC-MCC simulations, and the origin is placed at the center of the antenna at the interface between the metal wall and the plasma in the $z$-direction. A cylindrical coordinate system, where the radius $r$ is defined as $r=\sqrt{x^{2}+y^{2}}$ and the angle $\theta$ is set to $0^{\circ}$ and $270^{\circ}$ for $y$ - and $x$-axes, respectively, is also used to describe plasma distributions. The calculation domain consists of a $20 \times 20 \times 4 \mathrm{~mm}^{3}$ discharge chamber, a 0.6-mm-thick orifice plate with four circular orifices for electron extraction, and a $20 \times 20 \times 5.4 \mathrm{~mm}^{3}$ region to investigate the electron extraction in the outside region of the discharge chamber. As boundary conditions, the potential on the metal and boron nitride $(\mathrm{BN})$ was set to zero, and the potential at $z=10 \mathrm{~mm}$ was set to $20 \mathrm{~V}$ for the electron extraction voltage. This extraction voltage is the same as that used in our previous experiment, where a metal mesh plate was placed downstream of the neutralizer as an electron collector. ${ }^{21}$ The size of the plate was much larger than that of the neutralizer; thus, the extraction voltage was uniform along the radial direction. In addition, all 
(a)

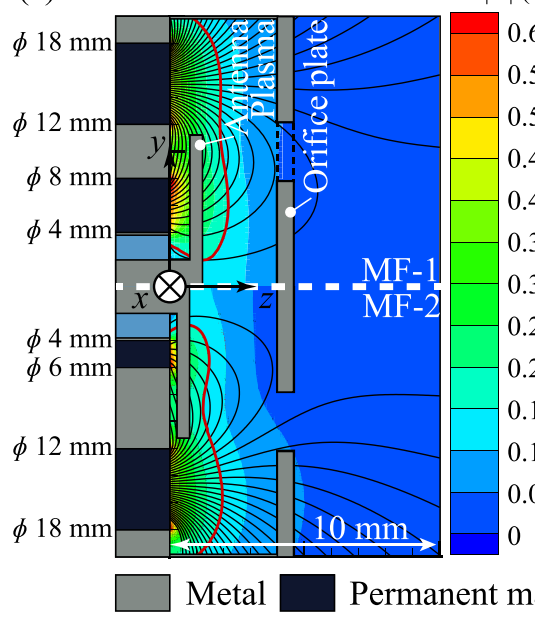

$|\mathrm{B}|(\mathrm{T})(\mathrm{b})$

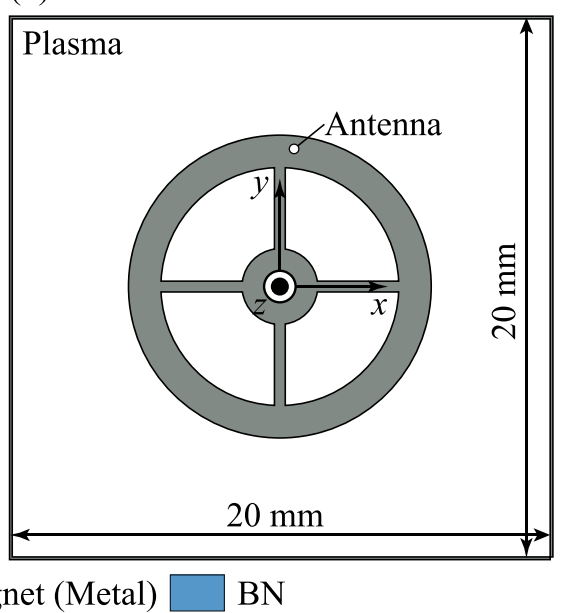

(c)

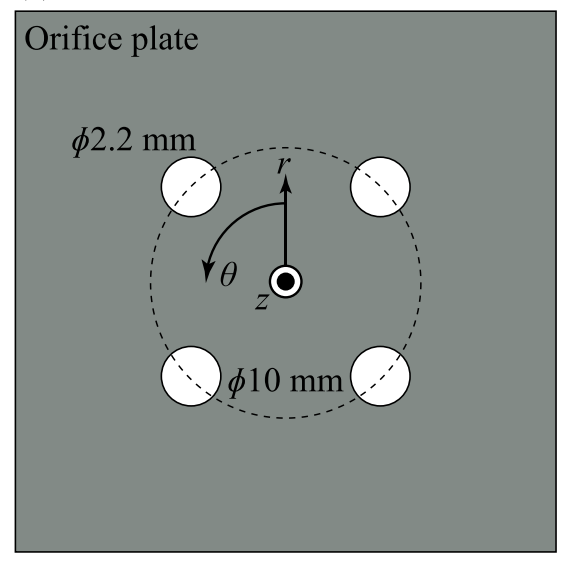

FIG. 2. Schematic diagram of the calculation model: (a) the $z-y$ plane $(x=0 \mathrm{~mm}$ ) together with the magnetic field for MF-1 (upper side) and MF-2 (lower side), (b) the $x-y$ plane $(z=1.0 \mathrm{~mm}$ for MF-1 and $z=0.5 \mathrm{~mm}$ for MF-2) at the antenna, and (c) the $x-y$ plane $(z=4.0 \mathrm{~mm})$ at the orifice plate. The thick red lines in panel (a) represent the resonant magnetic field of $0.15 \mathrm{~T}$, which is required to obtain ECR discharge heating for $4.2-\mathrm{GHz}$ microwaves.

electrons and ions disappeared at the wall, antenna, and other boundaries, where no reflection, secondary electron emission, or charge accumulation was assumed. Here, the ion energy at the boundaries was below a few tens of volts because the plasma source did not have any $\mathrm{dc}$ bias. Owing to the magnetic mirror configuration, the plasma density was negligibly small on the $\mathrm{BN}$ surface. Moreover, the $\mathrm{BN}$ area was considerably smaller compared with other surfaces $(0.94 \%$ of the total surface area excluding the antenna surface); thus, the effect of charge accumulation on the plasma potential was also negligible. The effect was already investigated for a micro inductively coupled plasma source in our previous study, where the voltage on the dielectric was only $1 \mathrm{~V}$ (see Figs. 7 and 10 in Ref. 22).

Table I shows calculation conditions for the water and xenon neutralizers, which are the same as those employed in our previous studies (see Refs. 15, 16, and 19 for xenon and Refs. 17 and 18 for water). The above-mentioned table shows that the microwave frequency was set to $4.2 \mathrm{GHz}$ for both neutralizers. However, the absorbed power and the neutral gas pressure of the water neutralizer were set to higher values than those of the xenon neutralizer to maintain the same order of plasma density as that of xenon. We assumed that neutral particles are spatially and temporally uniform with a Maxwellian velocity distribution at the gas temperature of $300 \mathrm{~K}$, which is the common assumption for low-pressure plasma sources. ${ }^{23-25}$ For the water neutralizer, $\mathrm{H}_{2} \mathrm{O}^{+}, \mathrm{OH}^{+}, \mathrm{H}^{+}, \mathrm{H}^{-}, \mathrm{O}^{-}$, $\mathrm{OH}^{-}$, and electrons were taken into account, whereas only $\mathrm{Xe}^{+}$and electrons were considered for the xenon neutralizer. The collision

TABLE I. Calculation conditions for the water and xenon neutralizers.

\begin{tabular}{lcc}
\hline \hline Propellant & $\mathrm{H}_{2} \mathrm{O}$ & $\mathrm{Xe}$ \\
\hline Microwave frequency & $4.2 \mathrm{GHz}$ & $4.2 \mathrm{GHz}$ \\
Absorbed power & $2.0 \mathrm{~W}$ & $0.3 \mathrm{~W}$ \\
Neutral gas pressure & $6.3 \mathrm{mTorr}$ & $1 \mathrm{mTorr}$ \\
\hline \hline
\end{tabular}

reactions and their cross sections for each neutralizer were the same as those employed in our previous papers (see Ref. 18 for water and Ref. 16 for xenon), where the null-collision method in MCC was used to reduce the calculation time. ${ }^{26}$ On the basis of the neutral gas pressures in Table I and the cross sections used in the simulations, the maximum frequency of electron- and ion-neutral collisions for the xenon and water neutralizers was calculated using the above-mentioned method. The calculation showed that the minimum mean-free time of the electron-neutral collision was $4.0 \times 10^{-8} \mathrm{~s}$ for xenon and $3.2 \times 10^{-9} \mathrm{~s}$ for water and that of the ion-neutral collision was $2.6 \times 10^{-6} \mathrm{~s}$ for xenon and $1.0 \times 10^{-6} \mathrm{~s}$ for water. The PIC-MCC time steps of $\Delta t_{\mathrm{e}}$ $=5.95 \times 10^{-12} \mathrm{~s}$ and $\Delta t_{\mathrm{i}}=2.38 \times 10^{-10} \mathrm{~s}$ are a few orders of magnitude lower than these values. Therefore, the collision of charged particles can be treated separately from the calculation of motion. ${ }^{2}$

\section{Additional remarks}

A previous study on the water plasma source revealed that the number density of the total negative ions was at least one order of magnitude smaller than that of electrons under the condition shown in Table I. ${ }^{18}$ This result indicates that the extracted negative ion currents have little effect on the neutralization performance of the water neutralizer. Hence, we took into account only electrons for the performance evaluation. The results of the simulations shown in the following section were averaged over 50000 microwave cycles $(11.9 \mu \mathrm{s})$ after the steady-state solutions were obtained. For 3D PIC-MCC simulations, the threads/cores were increased (up to 72/36) for the parallel computation to reduce the calculation time.

As mentioned in Sec. I, it is difficult to conduct plasma diagnostics for the spatial distribution of plasma parameters owing to the small size. The Langmuir probe method is very challenging owing to the strong magnetic field, as shown in Fig. 2(a), and probe insertion considerably perturbs the electromagnetic field of microwaves, which leads to completely different plasma profiles. Nonetheless, some comparisons between the numerical results and experimental data were conducted in our previous studies (see Refs. 16 and 21 for xenon and 
Ref. 17 for water), which are qualitatively in good agreement with each other.

\section{RESULTS AND DISCUSSION}

Figure 3 shows the time evolution of the volume-averaged electron number density $n_{\mathrm{e}}$, total positive ion density $n_{+}\left(\mathrm{H}_{2} \mathrm{O}^{+}, \mathrm{OH}^{+}\right.$, and $\left.\mathrm{H}^{+}\right)$, total negative ion density $n_{-}\left(\mathrm{OH}^{-}, \mathrm{O}^{-}\right.$, and $\left.\mathrm{H}^{-}\right)$for the water neutralizer together with the volume-averaged electron density $n_{\mathrm{e}}$, and positive ion density $n_{+}$for the xenon neutralizer in a time span of 50000 microwave cycles $(11.9 \mu \mathrm{s})$. Here, the value of $n_{-}$shown in Fig. 3 was multiplied by 10 . These values were averaged over the entire calculation domain. As shown in the figure, each density has reached a quasi-steady state, although there are some periodic oscillations attributed to plasma instability, as shown in our previous papers, ${ }^{15,18,19}$ which has been frequently observed in other $\boldsymbol{E} \times \boldsymbol{B}$ devices. ${ }^{28-32}$ The calculation results shown in Figs. 4-7 were averaged over the time span shown in Fig. 3.

\section{A. Electron extraction efficiency and loss}

Figure 4 shows the classification of the neutralizer walls and time-averaged fractions of the electron currents at each boundary, where the electron current toward the sidewall is not presented because it was negligibly small (less than $0.1 \%$ ). As shown in Fig. 4(b), the efficiency in MF-2 was increased by approximately two times (from $10 \%$ to 19\%) for the water neutralizer, which is the same trend as that for the xenon neutralizer [Fig 4(c)] but with a higher rate of increase. However, the electron extraction efficiency of the water neutralizer was still much lower than that of the xenon neutralizer $(49 \%$ in MF-2) owing to the high electron loss ratio inside the discharge chamber of the water neutralizer, where the electrons were dominantly lost to the upstream surface.

When MF-1 was applied to the neutralizer, the electron loss ratio at the upstream surface of the water neutralizer was approximately

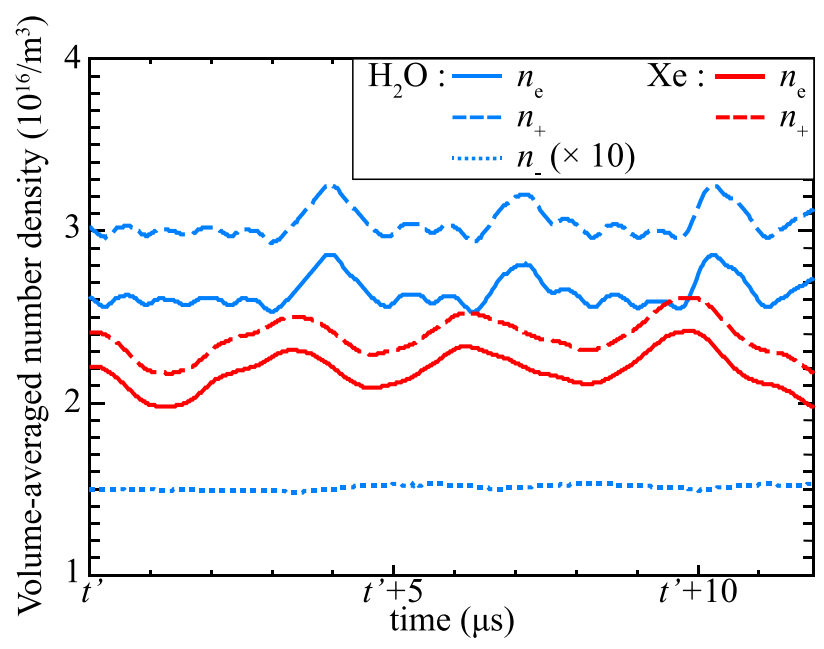

FIG. 3. Time evolution of the volume-averaged electron density (blue line), total positive ion density $\left(\mathrm{H}_{2} \mathrm{O}^{+}, \mathrm{OH}^{+}\right.$, and $\mathrm{H}^{+}$) (dashed blue line), and total negative ion density $\left(\mathrm{OH}^{-}, \mathrm{O}^{-}\right.$, and $\left.\mathrm{H}^{-}\right)$(dotted blue line) for the water neutralizer and that of the volume-averaged electron density (red line) and positive ion density (dashed red line) for the xenon neutralizer, which are averaged over the entire calculation domain. Here, the value of the total negative ion density was multiplied by 10 . (a)

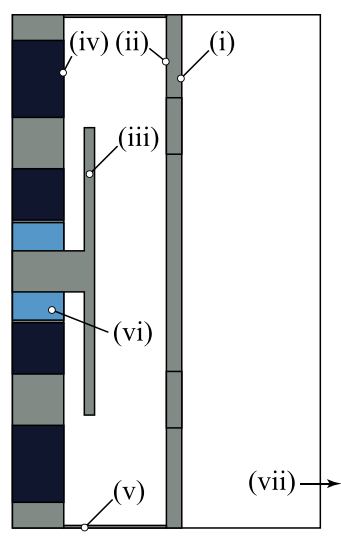

(b) $\mathrm{H}_{2} \mathrm{O}$

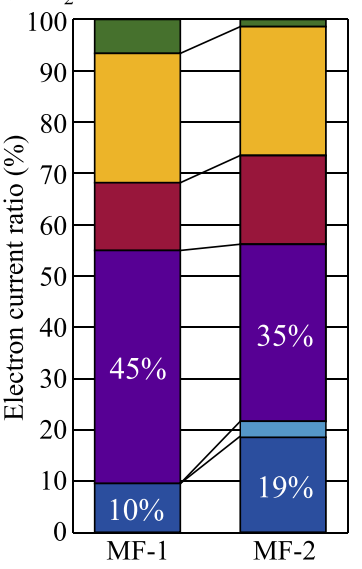

(c) $\mathrm{Xe}$

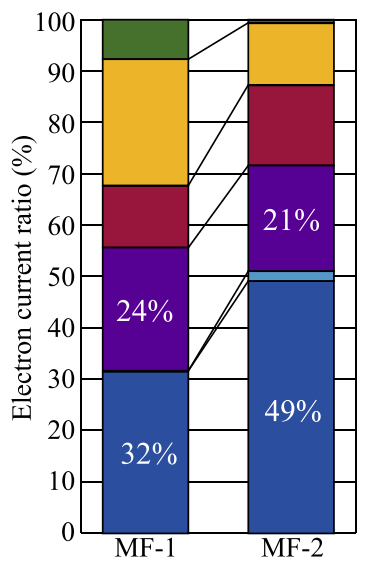

FIG. 4. (a) Classification of neutralizer walls and (b) time-averaged fractions of electron currents at each boundary for water and (c) those for xenon neutralizer. Here, the electron current toward the sidewall $(v)$ is not shown in panels $(b)$ and $(c)$ because it was negligibly small.

twice as high as that of the xenon neutralizer, whereas the electron loss ratios of other surfaces were approximately the same. Hence, we compared the simulation results of the water and xenon neutralizers for MF-1 by focusing on the electron loss to the upstream surface. By reducing the upstream loss, we could increase the electron extraction efficiency of the water neutralizer. The simulation results for MF-1 are shown in the remainder of this paper.

\section{B. Plasma distribution}

Next, we investigated plasma distributions of each neutralizer. Figure 5 shows the time-averaged distributions of the electron density, total positive ion density, total negative ion density, electron temperature, and potential on the $z-r$ plane at $\theta=45^{\circ}$ for the water (upper side) and xenon (lower side) neutralizers together with the ionization mean-free path. The black lines and dashed red lines in Fig. 5(a) represent the magnetic field lines and the resonant magnetic field of $0.15 \mathrm{~T}$ to obtain ECR discharge heating for 4.2-GHz microwaves, respectively. As shown in Fig. 5(a), a high electron density region appeared along the ECR layer, where the order of the peak electron density in both 
(a)

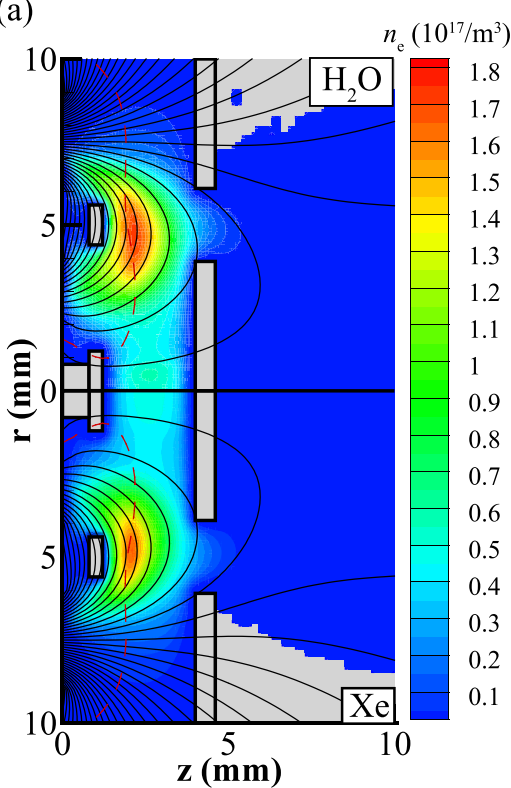

(d)

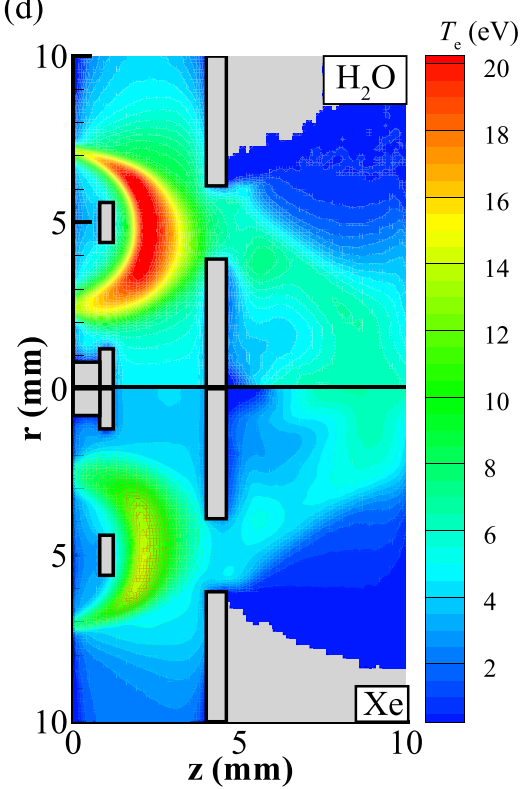

(b)

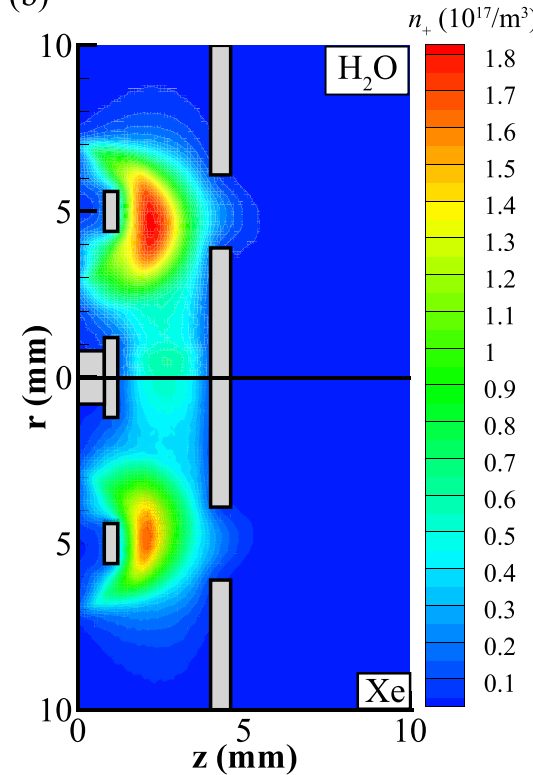

(e)

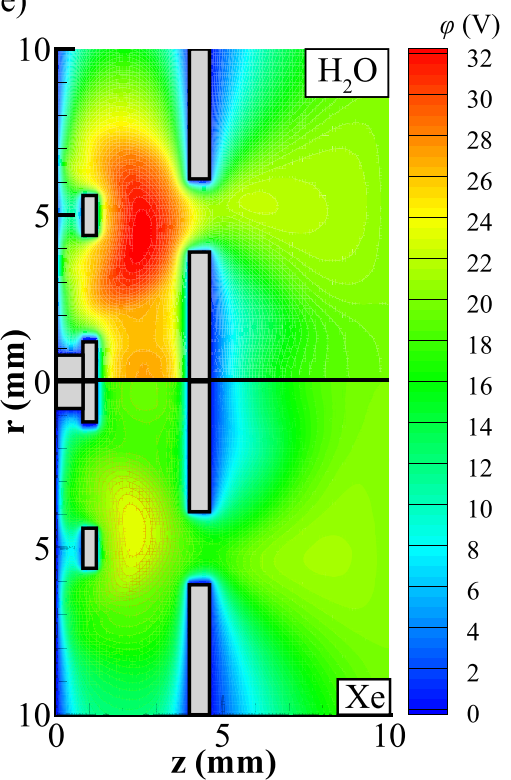

(c)

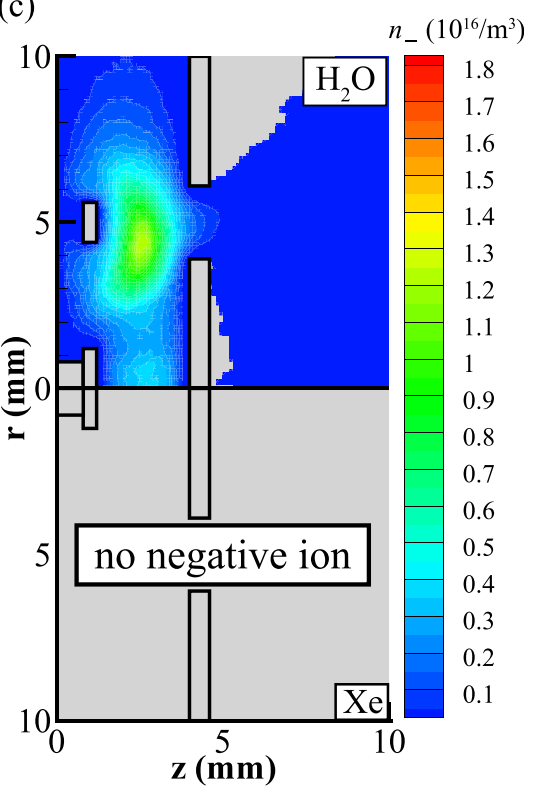

(f)

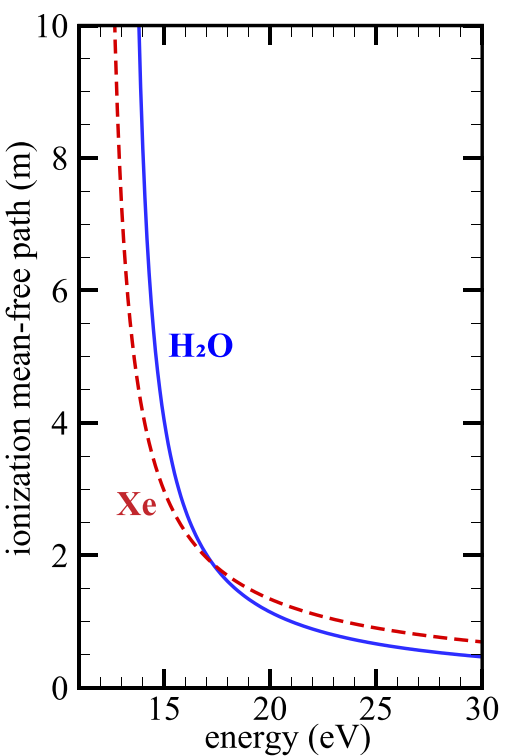

FIG. 5. Time-averaged distributions of the (a) electron density, (b) total positive ion density, (c) total negative ion density, (d) electron temperature, and (e) potential of the water (upper side) and xenon (lower side) neutralizers for MF-1, where the black and dashed red lines in panel (a) represent the magnetic field lines and the resonant magnetic field of $0.15 \mathrm{~T}$ to obtain ECR discharge heating for $4.2-\mathrm{GHz}$ microwaves, respectively. The distribution at the $z-r$ plane $\left(\theta=45^{\circ}\right)$ is shown, where the $y$-axis is drawn at $0^{\circ}$ and the $x$-axis is drawn at $270^{\circ}$. (f) lonization mean-free path as a function of electron energy for water (blue line) and xenon (dashed red line).

neutralizers was the same because the absorbed power and the neutral gas pressure of the water neutralizer were increased, as mentioned in Sec. II B. The distribution of the positive ion density in both neutralizers shown in Fig. 5(b) was approximately the same as that of the electron density. However, negative ions, which existed only in the water neutralizer, were confined by the plasma potential, as shown in Figs. 5(c) and 5(e). The density of the negative ions was less than a tenth of the electron and positive ion densities. These distributions indicated that quasineutrality was confirmed in both water and xenon plasma sources.

However, as shown in Fig. 5(d), the electron temperature of the water neutralizer was higher than that of the xenon neutralizer. The threshold energy of ionization for water was higher than that for xenon. Moreover, as shown in Fig. 5(f), the ionization mean-free path of low energy electrons $(<17.5 \mathrm{eV})$ in the water neutralizer was larger 

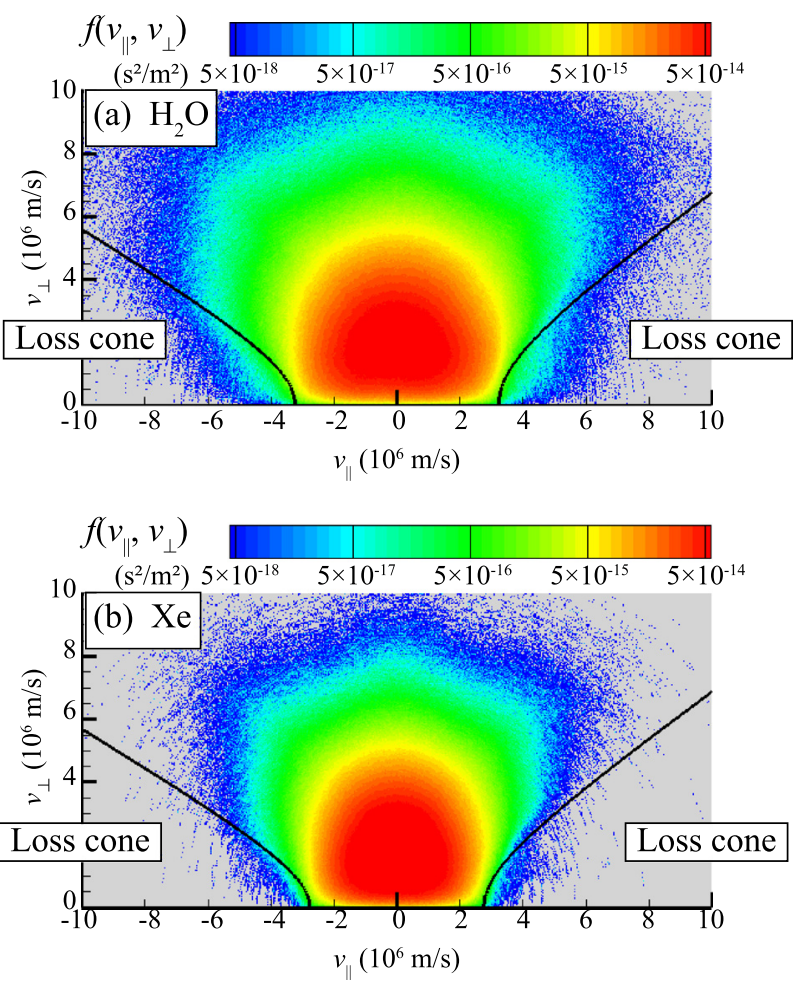

FIG. 6. Normalized electron velocity distribution functions $f\left(v_{\|}, v_{\perp}\right)$ at $(x, y, z)$ $=(0 \mathrm{~mm}, 5.0 \mathrm{~mm}, 2.0 \mathrm{~mm})$ for $(\mathrm{a})$ the water and (b) xenon neutralizers. Thick black lines represent the boundary of the loss cone obtained from Eq. (2).

than that in the xenon neutralizer; although the neutral gas pressure of the water neutralizer was higher than that of the xenon neutralizer. Owing to the longer mean-free path and higher absorbed power, as shown in Table I, the electron temperature of the water neutralizer reached a higher value than that of the xenon neutralizer. The potential of the water neutralizer also became high owing to the high electron temperature [Fig. 5(e)].

Because the peak plasma potential in both water and xenon plasma sources was higher than the extraction voltage of $20 \mathrm{~V}$ at $z=10 \mathrm{~mm}$, ions were distributed in the entire extraction region (outside the plasma source), as shown in Fig. 5(b). The extracted electrons were also confined by the magnetic field in the extraction region
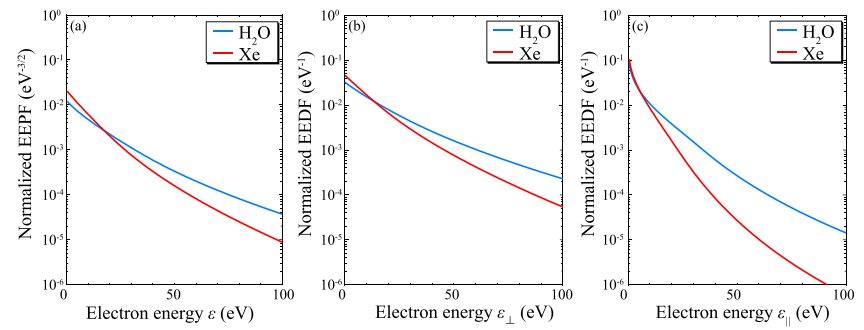

FIG. 7. (a) Normalized EEPF $g_{p}(\varepsilon)$, (b) normalized EEDF in the perpendicular direction to the magnetic field $g_{\mathrm{d}}\left(\varepsilon_{\perp}\right)$, and (c) that in the parallel direction $g_{\mathrm{d}}\left(\varepsilon_{\|}\right)$in the peak plasma region $(1.5 \mathrm{~mm} \leq z \leq 3.5 \mathrm{~mm}$ and $3 \mathrm{~mm} \leq r \leq 6 \mathrm{~mm})$ for the water and xenon neutralizers.
[Fig. 5(a)]. Here, both the ion and electron densities outside the plasma sources were in the same range of $10^{13}-10^{15} \mathrm{~m}^{-3}$.

In the plasma sources, electrons seemed to be confined along the magnetic fields, which indicated that the magnetic mirror trapped most electrons. Thus, the electrons that are present within the loss cone will be lost to permanent magnets placed at the upstream surface. This mechanism is discussed in the following section.

\section{Loss cone of the magnetic mirror}

The ratio of electrons inside the loss cone can be evaluated by the normalized electron velocity distribution function $f\left(v_{\|}, v_{\perp}\right)$, where $v_{\|}$ represents the electron velocity component in the parallel direction to the magnetic field, and $v_{\perp}$ represents that in the perpendicular direction. ${ }^{33}$ We calculated $f\left(v_{\|}, v_{\perp}\right)$ at the position where nearly the peak plasma density was observed $(x=0 \mathrm{~mm}, y=5.0 \mathrm{~mm}$, and $z=2.0 \mathrm{~mm}$ ). In the velocity phase space, the following relation for the trapped electron is derived: ${ }^{3}$

$$
v_{0, \|}< \pm \sqrt{\left(\frac{B_{1}}{B_{0}}-1\right) v_{0, \perp}^{2}-2 q \frac{\left(\phi_{0}-\phi_{1}\right)}{m_{\mathrm{e}}}},
$$

where $m_{\mathrm{e}}$ is the electron mass, $\phi$ is the potential, $q$ is the charge of the electron, $B$ is the magnetic field, subscript " 0 " represents the value at the position where $f\left(v_{\|}, v_{\perp}\right)$ was calculated, and subscript " 1 " represents the value at the position where the magnetic mirror reflected electrons. In the water and xenon neutralizers, $\phi_{1}$ in Eq. (1) represents the potential of $0 \mathrm{~V}$ at the upstream surface, and $B_{1}$ represents the magnetic field at the electron loss position. Note that $B_{1}$ of the inner and outer ring-shaped magnets are different in the neutralizer, as shown in Fig. 2(a). Hence, the relation for the trapped electron in the neutralizer is given as follows:

$$
-\sqrt{\left(\frac{B_{\text {in }}}{B_{0}}-1\right) v_{0, \perp}^{2}-\frac{2 q \phi_{0}}{m_{\mathrm{e}}}}<v_{0, \|}<\sqrt{\left(\frac{B_{\text {out }}}{B_{0}}-1\right) v_{0, \perp}^{2}-\frac{2 q \phi_{0}}{m_{\mathrm{e}}}},
$$

where $B_{\text {in }}$ and $B_{\text {out }}$ represent the magnetic field at the electron loss position on the inner and outer magnets, respectively. Using Eq. (2), we evaluated the electron loss toward the upstream surface.

Figure 6 shows the distributions of $f\left(v_{\|}, v_{\perp}\right)$ for the water and xenon neutralizers. These distributions were calculated at the position of $(x, y, z)=(0 \mathrm{~mm}, 5.0 \mathrm{~mm}, 2.0 \mathrm{~mm})$, where nearly the peak plasma density was observed, as mentioned earlier. Here, the thick black lines represent the boundary obtained from Eq. (2). The electrons, which did not satisfy Eq. (2), were in the loss cone and, thus, lost to the upstream surface if the electrons did not collide with the other particles or the antenna. As shown in Fig. $6, f\left(v_{\|}, v_{\perp}\right)$ of the water neutralizer was wider in both $v_{\|}$and $v_{\perp}$ directions than that of the xenon neutralizer owing to the higher electron temperature. Thus, the ratio of the number of electrons inside the loss cone to that of all electrons in the velocity phase space was calculated to be $0.11 \%$ for the water neutralizer, which was approximately 5 -times higher than that for the xenon neutralizer $(0.023 \%)$.

In addition, we calculated the electron energy probability function (EEPF) and the electron energy distribution function (EEDF) in the peak plasma region $(1.5 \mathrm{~mm} \leq z \leq 3.5 \mathrm{~mm}$ and $3 \mathrm{~mm} \leq r \leq 6 \mathrm{~mm}$ ). Here, the definition of EEPF is the same as that in previous 
papers. ${ }^{16,22,35}$ Figure 7 shows the normalized EEPF $g_{\mathrm{p}}(\varepsilon)$, normalized EEDF in the perpendicular direction to the magnetic field $g_{\mathrm{d}}\left(\varepsilon_{\perp}\right)$, and that in the parallel direction $g_{\mathrm{d}}\left(\varepsilon_{\|}\right)$. Figure 7 (a) shows that the electron temperature evaluated by the gradient of $g_{\mathrm{p}}(\varepsilon)$ was $13-22 \mathrm{eV}$ for the water neutralizer and $10-17 \mathrm{eV}$ for the xenon neutralizer, which agreed with the values in Fig. 5(d). As shown in Figs. 7(b) and 7(c), the ratio of high energy electrons $(>10-15 \mathrm{eV})$ in the water neutralizer was larger than that in the xenon neutralizer in both perpendicular and parallel directions. However, the difference in $g_{\mathrm{d}}\left(\varepsilon_{\|}\right)$for water and xenon neutralizers was larger than the difference in $g_{\mathrm{d}}\left(\varepsilon_{\perp}\right)$ for water and xenon neutralizers, indicating that the ratio of $v_{\|}$to $v_{\perp}$ in the water neutralizer was higher than that in the xenon neutralizer. Hence, the number of electrons inside the loss cone of the water neutralizer was larger than that of the xenon neutralizer, which agreed with the result obtained from Fig. 6. An increase in the electron loss to the upstream surface is related to the loss cone of the magnetic mirror.

ECR heating accelerates only $\boldsymbol{v}_{\perp}$ of electrons, which results in higher $\boldsymbol{v}_{\perp}$ than $\boldsymbol{v}_{\|}$. Therefore, as shown in Eq. (2), the number of electrons trapped by the magnetic mirror increases owing to ECR heating. However, when electrons collide with other particles, their velocity components are changed irregularly, and the difference between $\boldsymbol{v}_{\perp}$ and $\boldsymbol{v}_{\|}$becomes smaller. Therefore, with an increase in the number of electron collisions, the number of electrons within the loss cone tends to increase. As mentioned in Sec. II B, the neutral gas pressure in the water neutralizer was set to the higher value than that of the xenon neutralizer. Therefore, the high neutral gas pressure in the water neutralizer led to a number of electron collisions. Thus, the number of electrons present within the loss cone in the water neutralizer was larger than that in the xenon one. The result implies that a decrease in the neutral pressure may reduce the electron loss to the upstream surface and increase the electron extraction current. However, it was not possible to increase the extraction current at lower pressures owing to a decrease in the electron density. Because the neutralizer configuration was originally developed for the xenon propellant, a new chamber design may be required for the water neutralizer to reduce the electron loss and to increase the extraction current; this work will be performed in the future.

\section{CONCLUSIONS}

This study presented the 3D PIC-MCC simulations of a $4.2-\mathrm{GHz}$ water ECR neutralizer to elucidate the electron loss mechanisms toward the upstream surface, where two ring-shaped permanent magnets are placed for the ECR discharge and electron confinement by the magnetic mirror. We conducted simulations for the two magnetic field configurations of MF-1 and MF-2. MF-1 is the conventional magnetic field, and MF-2 is the optimized magnetic field, where the performance of the xenon neutralizer was improved in the previous study. ${ }^{15}$ Here, the neutralizer performance was evaluated by the electron extraction efficiency, which is defined as the ratio of the extracted electron current to the generated electron current.

The simulation results for the water neutralizer showed that MF2 led to an increase in the electron extraction efficiency by approximately two times. However, it was also confirmed that the extraction efficiency of the water neutralizer was lower than that of the xenon neutralizer because the electron loss to the upstream surface of the water neutralizer was much higher than that of the xenon neutralizer, especially for MF-1. The result implied that the number of electrons inside the loss cone for the water neutralizer was larger than that for the xenon one.

The ratio of the electrons present within the loss cone was also evaluated by the electron velocity/energy distribution functions in the peak plasma region. The number of electrons inside the loss cone of the water neutralizer was determined to be approximately 5-times larger than that of the xenon neutralizer. Therefore, we concluded that an increase in the electron loss toward the upstream surface for the water neutralizer was attributed to the larger number of electrons present within the loss cone. This mechanism was probably caused by the higher collision frequency owing to the higher neutral pressure of the water neutralizer, which decreased the difference between the electron velocity in the parallel direction to the magnetic field and that in the perpendicular direction. Future work will include an investigation on the ways to reduce electron loss toward the upstream surface to increase the electron extraction efficiency of the water neutralizer.

\section{ACKNOWLEDGMENTS}

This work was partially supported by JSPS KAKENHI, Grant No. JP16H06370. It partly used computational resources under the Collaborative Research Project for Enhancing Performance of Programming by the Academic Center for Computing and Media Studies, Kyoto University. Part of the computer simulation was performed on a supercomputer at the KDK computer system at the Research Institute for Sustainable Humanosphere, Kyoto University.

\section{DATA AVAILABILITY}

The data that support the findings of this study are available from the corresponding author upon reasonable request.

\section{REFERENCES}

${ }^{1}$ A. T. Sugiarto and M. Sato, Thin Solid Films 386, 295 (2001).

${ }^{2}$ M. Sato, Plasma Sources Sci. Technol. 17, 024021 (2008).

${ }^{3}$ J. Oh, K. Kawamura, B. K. Pramanik, and A. Hatta, IEEE Trans. Plasma Sci. 37, 107 (2009).

${ }^{4}$ B. Sun, M. Sato, and J. S. Clements, Environ. Sci. Technol. 34, 509 (2000).

${ }^{5}$ S. V. T. Nguyen, J. E. Foster, and A. D. Gallimore, Rev. Sci. Instrum. 80, 083503 (2009)

${ }^{6}$ R. A. Rudder, G. C. Hudson, J. B. Posthill, R. E. Thomas, R. C. Hendry, D. P. Malta, R. J. Markunas, T. P. Humphreys, and R. J. Nemanich, Appl. Phys. Lett. 60, 329 (1992).

${ }^{7^{7}}$. Koizumi, Y. Kawazoe, K. Komurasaki, and Y. Arakawa, Vacuum 80, 1234 (2006).

${ }^{8}$ E. M. Petro and R. J. Sedwick, J. Propul. Power 33, 1410 (2017).

${ }^{9}$ J. E. Brandenburg, J. Kline, and D. Sullivan, IEEE Trans. Plasma Sci. 33, 776 (2005).

${ }^{10}$ A. Poghosyan and A. Golkar, Prog. Aerosp. Sci. 88, 59 (2017).

${ }^{11}$ Y. Nakagawa, D. Tomita, H. Koizumi, and K. Komurasaki, Trans. Jpn. Soc. Aeronaut. Space Sci. Aerosp. Technol. Jpn. 16, 673 (2018).

${ }^{12}$ Y. Nakagawa, H. Koizumi, H. Kawahara, and K. Komurasaki, Acta Astronaut. 157, 294 (2019).

${ }^{13}$ H. Koizumi, K. Komurasaki, J. Aoyama, and K. Yamaguchi, Trans. Jpn. Soc. Aeronaut. Space Sci. Aerosp. Technol. Jpn. 12, Tb_19 (2014).

${ }^{14}$ H. Koizumi, H. Kawahara, K. Yaginuma, J. Asakawa, Y. Nakagawa, Y. Nakamura, S. Kojima, T. Matsuguma, R. Funase, J. Nakatsuka, and K. Komurasaki, Trans. Jpn. Soc. Aeronaut. Space Sci. Aerosp. Technol. Jpn. 14, Pb_13 (2016).

${ }^{15}$ Y. Sato, H. Koizumi, M. Nakano, and Y. Takao, J. Appl. Phys. 126, 243302 (2019). 
${ }^{16}$ Y. Takao, H. Koizumi, K. Komurasaki, K. Eriguchi, and K. Ono, Plasma Sources Sci. Technol. 23, 064004 (2014).

${ }^{17}$ K. Nakamura, Y. Nakagawa, H. Koizumi, and Y. Takao, Trans. Jpn. Soc. Aeronaut. Space Sci. 61, 152 (2018).

${ }^{18}$ K. Nakamura, H. Koizumi, M. Nakano, and Y. Takao, Phys. Plasmas 26, 043508 (2019)

${ }^{19}$ K. Hiramoto, Y. Nakagawa, H. Koizumi, and Y. Takao, Phys. Plasmas 24, 064504 (2017)

${ }^{20}$ C. K. Birdsall and A. B. Langdon, Plasma Physics via Computer Simulation (IOP Publishing, Bristol, UK, 1991).

${ }^{21}$ Y. Takao, H. Koizumi, Y. Kasagi, and K. Komurasaki, Trans. Jpn. Soc. Aeronaut. Space Sci. Aerosp. Technol. Jpn. 14, Pb_41 (2016).

${ }^{22}$ Y. Takao, K. Eriguchi, and K. Ono, J. Appl. Phys. 112, 093306 (2012).

${ }^{23}$ E. Kawamura, M. A. Lieberman, A. J. Lichtenberg, and P. Chabert, J. Vacuum Sci. Technol., A 38, 023003 (2020).

${ }^{24}$ F. Taccogna, P. Minelli, Z. Asadi, and G. Bogopolsky, Plasma Sources Sci. Technol. 28, 064002 (2019).
${ }^{25}$ S. L. Guo, X. L. Jin, L. Lei, X. Y. Zhang, X. F. Zhu, Z. H. Yang, and B. Li, Phys. Plasmas 26, 073502 (2019).

${ }^{26}$ V. Vahedi and M. Surendra, Comput. Phys. Commun. 87, 179 (1995).

${ }^{27}$ K. Nanbu, IEEE Trans. Plasma Sci. 28, 971 (2000).

${ }^{28}$ M. Panjan and A. Anders, J. Appl. Phys. 121, 063302 (2017).

${ }^{29}$ T. Lafleur, S. D. Baalrud, and P. Chabert, Plasma Sources Sci. Technol. 26, 024008 (2017).

${ }^{30}$ M. Keidar, I. D. Boyd, and I. I. Beilis, Phys. Plasmas 8, 5315 (2001).

${ }^{31}$ T. Lafleur, R. Martorelli, P. Chabert, and A. Bourdon, Phys. Plasmas 25, 061202 (2018).

${ }^{32}$ A. Hecimovic, J. Phys. D 49, 18LT01 (2016).

${ }^{33}$ X. Liu, L. Chen, W. Gu, and X.-J. Zhang, J. Geophys. Res. Space 123, 9035, https://doi.org/10.1029/2018JA025925 (2018).

${ }^{34}$ F. H. Ebersohn, J. P. Sheehan, A. D. Gallimore, and J. V. Shebalin, J. Comput. Phys. 351, 358 (2017).

${ }^{35}$ Y. Takao, N. Kusaba, K. Eriguchi, and K. Ono, J. Appl. Phys. 108, 093309 (2010). 